Ann. Génét. Sél. anim., I973, 5 (2), 217-224.

\title{
CORRÉLATIONS ENTRE LE DIMORPHISME SEXUEL POUR LE POIDS ET D'AUTRES GARACTÈRES QUANTITATIFS CHEZ LA POULE
}

\author{
H. AYOUB et P. MÉRAT \\ Faculté d'A griculture, Université Ain-Shams, Le Caire, Égypte, \\ Laboratoive de Génétique factorielle, \\ Centre national de Recherches zootechniques, I. N. R. A., \\ 78350 Jouy en Josas
}

\section{RÉSUMÉ}

Dans deux populations distinctes, nous avons évalué les corrélations phénotypiques et génétiques entre dimorphisme sexuel du poids (différence entre moyenne des $\hat{o} \widehat{o}$ et moyenne des 우우) à 8 semaines et à l'âge adulte, par familles de frères-sœurs, et d'autres caractères d'intérêt économique. Une corrélation significative positive avec l'âge au $\mathrm{I}^{\mathrm{er}}$ œuf et négative avec la ponte d'automne et d'hiver est trouvée pour le dimorphisme du poids adulte; elle est confirmée par la corrélation partielle entre le poids des mâles et ces deux caractères, à poids des femelles fixé. Il serait souhaitable de savoir si ce phénomène existe dans d'autres populations. Dans ce cas, il serait utilisable dans la sélection pour la ponte.

Par ailleurs, le dimorphisme sexuel du poids présente une corrélation positive importante avec le poids des mâles, et une corrélation faiblement négative avec le poids des femelles, mais ces corrélations ne représentent pas par elles-mêmes un phénomène biologique réellement indépendant.

\section{INTRODUCTIGN}

Seu1 à notre connaissance, BuvanENDRAN (I969) a abordé le sujet des corrélations génétiques entre le dimorphisme sexuel et d'autres caractères quantitatifs importants du point de vue économique chez la poule. Sur une souche de Leghorns blanches non sélectionnée, la différence de poids à io semaines entre les deux sexes est déterminée pour chaque famille de frères-sœurs, en retranchant la moyenne des descendants femelles de celle des mâles. Les corrélations génétiques estimées entre 
ce dimorphisme sexuel et le poids des mâles au même âge, le poids des femelles, le poids des œufs ou le nombre d'œufs sont respectivement $+0,69 ;+0,06 ;+0,59$; +0, I4. On remarque donc une corrélation assez élevée avec le poids des mâles d'une part, le poids moyen des œufs de l'autre.

Dans un ordre d'idées analogue, KINNEy et SHOFFNER (I965) ont estimé les corrélations génétiques entre le poids des mâles à 8 semaines et le taux de ponte ou l'âge au I er œuf de leurs sœurs, dans une souche "chair " et une souche " ponte ". Dans la première, les mâles les plus lourds à 8 semaines ont les sœurs les plus précoces, mais non dans la seconde.

Nous avons recueilli des données provenant de deux origines relativement à ce problème, étant donné son intérêt possible, tant dans les souches " ponte " que dans la sélection " chair ".

\section{MATÉRIEL, ET MÉTHODES}

Le matériel utilisé est constitué d'abord par quatre années (1964 à 1967) du troupeau de Jouyen-Josas. Il s'agit d'une souche reproduite en troupeau fermé depuis I955, chaque mâle étant accouplé à 8-Io femelles et chaque femelle à un seul mâle. Les différentes familles sont élevées ensemble dans les mêmes locaux au sol, les deux sexes étant mélangés jusqu'à ro semaines d'âge.

Nous y étudions les corrélations phénotypiques, sur une base intra-années, entre le dimorphisme sexuel du poids à 8 semaines (différence moyenne de poids entre les deux sexes dans une famille de frères-sœurs) et les 5 caractères suivants :

- nombre d'œufs pondus à 1'âge de 44 semaines ;

- poids moyen des œufs au même âge (moyenne des 15 derniers jours) ;

- âge à la ponte du I ${ }^{\text {er }} œ u f$;

- poids des femelles à 8 semaines;

- poids des mâles à 8 semaines.

Nous avons aussi évalué la corrélation phénotypique entre le poids moyen des mâles et celui des femelles au même âge, dans chaque famille de frères-sœurs.

Concernant les souches du Magnevaud, nous avons eu la possibilité d'étudier le dimorphisme sexuel par familles de frères-sœurs à l'âge de 8 semaines, mais aussi à l'âge adulte, pour les années I 966 et I 967 de la souche M I I (Wyandotte blanche), où aucune élimination volontaire de mâles n'avait été faite jusqu'à l'âge adulte. L'année 1966 comporte 233 familles de mères et l'année I 967 en comporte 133 .

Les caractères étudiés dans cette souche en corrélation avec le dimorphisme sexuel à l'un ou l'autre âge sont les suivants :

- Age au I er œuf.

- Intensité de la ponte d'automne et d'hiver (de l'entrée en ponte au 3I-I2).

- Pourcentage d'éclosion par rapport aux œufs fertiles.

- Pourcentage de fertiles par rapport aux œufs mis en incubation.

- Poids des mâles à l'âge correspondant.

- Poids des femelles.

Nous avons, d'autre part, estimé la corrélation phénotypique entre le dimorphisme sexuel à chacun des deux âges mentionnés.

Les corrélations phénotypiques portent sur des moyennes par familles de frères-sœurs, moyennes qui ne sont pas strictement indépendantes, en particulier du fait de l'influence du père ; ceci ne devrait pas enlever leur signification aux corrélations estimées, étant donnéle nombre total assez grand des familles. D'autre part, nous n'avons pas tenu compte des familles dont l'effectif, dans l'un ou l'autre sexe, était inférieur à 5, pour que les moyennes ne soient pas établies sur des effectifs trop variables.

En complément, nous avons estimé sur le même matériel la corrélation partielle entre poids moyen des mâles aux âges indiqués et nombre d'œufs ou âge au I ${ }^{\text {er }}$ œuf de leurs sœurs à poids moyen fixé pour ces femelles.

Sur une partie des données, enfin, nous avons tenté d'évaluer une corrélation génétique entre dimorphisme sexuel et caractères de ponte. 


\section{RÉSULTATS}

Le tableau I montre les corrélations phénotypiques entre le dimorphisme sexuel à l'âge de 8 semaines et les autres caractères sur les données de Jouy.

Avec le nombre d'œufs, les corrélations sont faibles, variant entre $-0,2 I$ et $+0,15$. La corrélation globale intra-années est égale à $+0,007$, non significative.

Avec l'âge au I ${ }^{\text {er }}$ œuf, les corrélations par année varient entre $-0,36$ et $+0,03$. Quoique l'année 1965 présente une corrélation négative significative, la corrélation globale intra-année est très faible, égale à - o,o6.

Le poids des œufs présente une corrélation positive, mais faible, avec le dimorphisme.

Seule la corrélation avec le poids des mâles est toujours nettement positive et hautement significative, celle avec le poids des femelles étant faible.

Quant aux corrélations phénotypiques entre le poids des femelles et celui des mâles, elles sont toujours positives et hautement significatives dans tous les cas.

Le tableau 2 présente les résultats du Magneraud (souche M II) aux deux âges, 8 semaines et adulte.

Les corrélations avec le dimorphisme sexuel à l'âge de 8 semaines, comme sur les données de Jouy, ne sont appréciables et significatives qu'avec le poids des mâles.

La corrélation entre poids des femelles et poids des mâles est hautement significative, à cet âge, dans les deux années étudiées.

\section{TABLEAU 2}

Corrélation phénotypique entre le dimorphisme sexuel à 8 semaines ou à l'âge adulte et $\mathrm{y}$ caractères (souche M Ir du Magneraud)

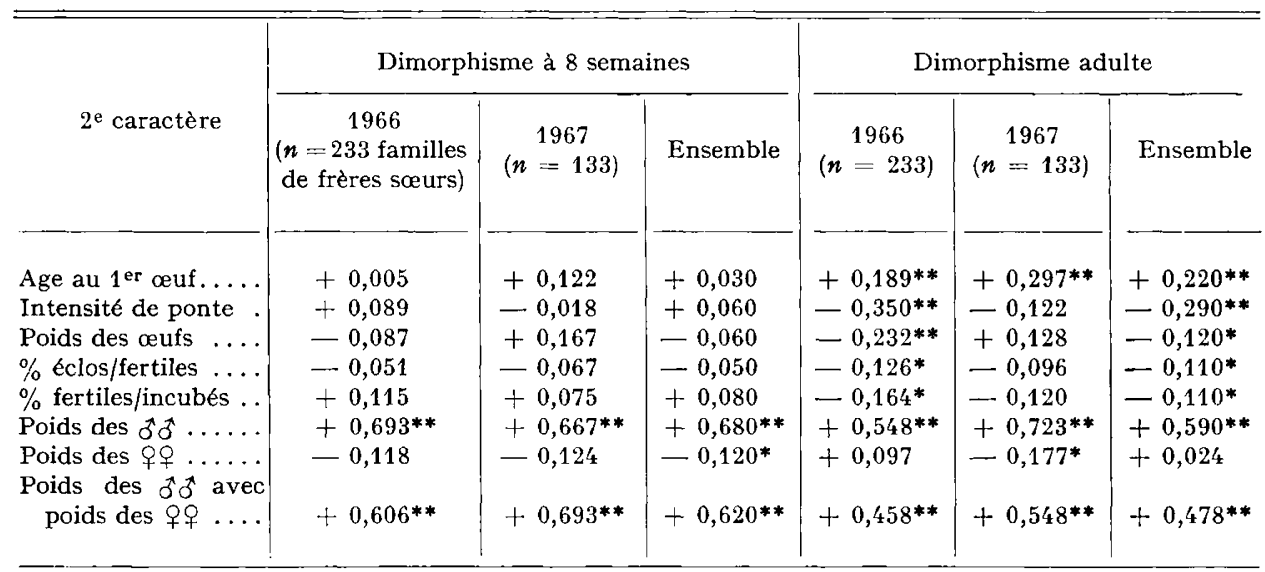

* Significatif au seuil 5 p. 100.

** Significatif au seuil 1 p. 100. 


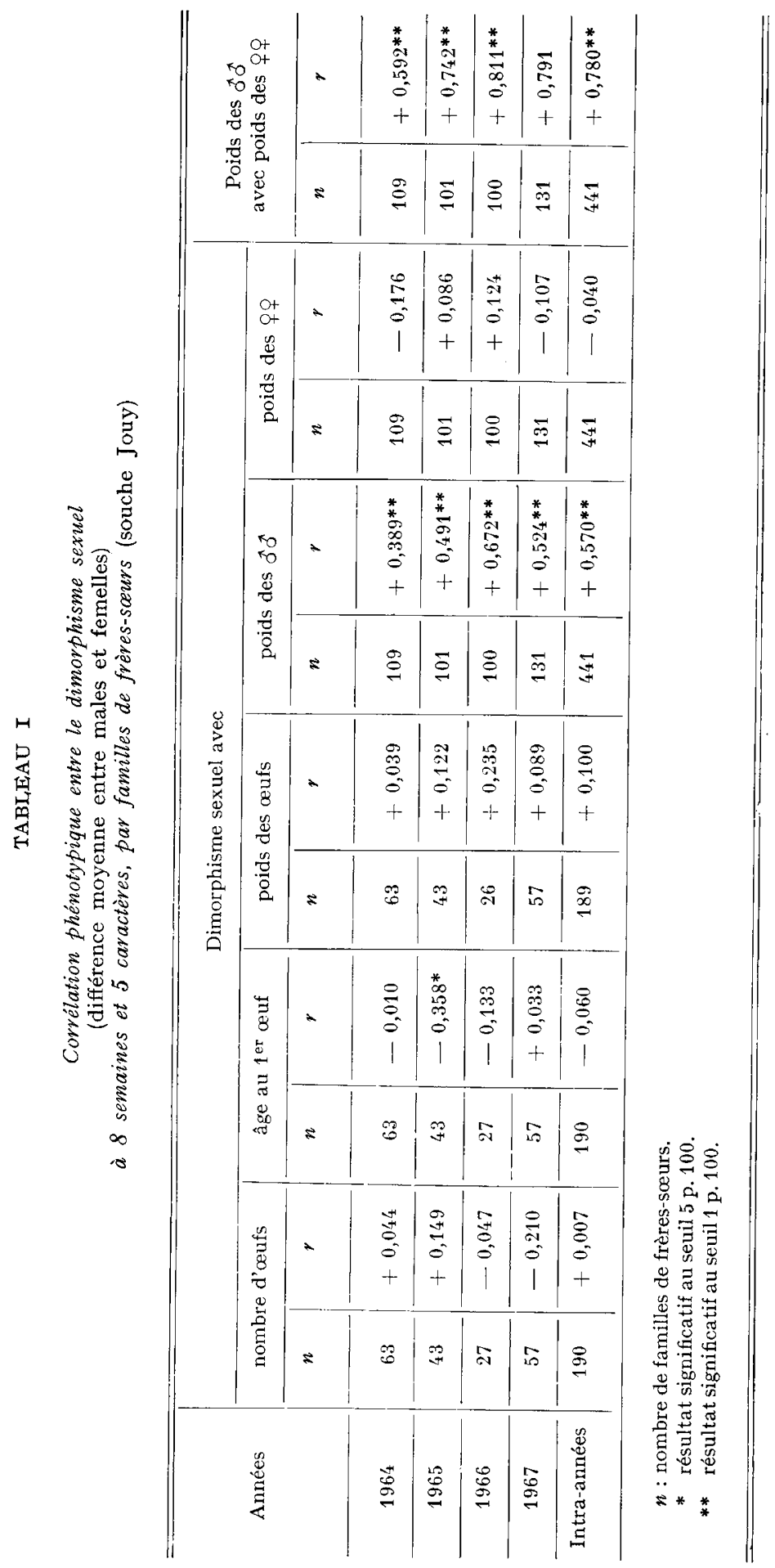


Le dimorphisme sexuel du poids adulte, par contre, montre :

- une corrélation positive avec l'âge au $\mathrm{I}^{\mathrm{er}}$ œuf de $+0,22(\mathrm{P}<0, \mathrm{OI})$

- une corrélation négative avec l'intensité de ponte de $-0,29(\mathrm{P}<0$,oI $)$

- une corrélation négative avec le poids des œufs de - 0,I2 $(\mathrm{P}<0,05)$

- une corrélation négative avec les taux de fertilité et d'éclosion.

La corrélation avec le poids des femelles est négligeable, mais celle avec le poids des mâles est importante, comme dans les cas précédents.

Les corrélations entre le poids des mâles et le poids des femelles sont aussi positives et hautement significatives.

A la suite de ces résultats, nous avons calculé parallèlement, sur les données correspondantes, les corrélations partielles entre nombre d'œufs et âge au I er œuf d'une part, dimorphisme sexuel dı poids aux deux âges étudiés d'autre part, à poids des femelles fixé (tabl. 3 et 4 ).

\section{TABLEAU 3}

Corrélation partielle du dimorphisme sexuel à 8 semaines (souche Jouy) avec deux caractères de ponte, à poids à 8 semaines fixé pour les 9 우 (par familles de frères-sœurs)

\begin{tabular}{|c|c|c|}
\hline \multirow{2}{*}{ Année } & \multicolumn{2}{|c|}{ Dimorphisme à 8 semaines avec } \\
\hline & Nombre d'œufs & Age au $1^{\text {er }} œ u f\left({ }^{1}\right)$ \\
\hline 1964 & $+0,32$ & $-0,35^{* *}$ \\
\hline 1965 & $+0,16$ & $-0,57 * *$ \\
\hline 1966 & $-0,03$ & $+0,09$ \\
\hline 1967 & $\longrightarrow 0,11$ & $+0,17$ \\
\hline $\begin{array}{c}\text { Total } \\
\text { (Intra-années) }\end{array}$ & $+0,13^{*}$ & $-0,22^{* *}$ \\
\hline
\end{tabular}

(1) La même corrélation pour 1969 est égale à $-0,36$ **.

TABLEAU 4

Corrélation partielle du dimorphisme sexuel à 8 semaines et adulte (souche M II) avec deux caractères de ponte, à poids fixé pour les +9 (par familles de frères-sœurs)

\begin{tabular}{|c|c|c|c|c|}
\hline \multirow[t]{2}{*}{ Année } & \multicolumn{2}{|c|}{$\begin{array}{l}\text { Dimorphisme à } 8 \text { semaines } \\
\text { avec }\end{array}$} & \multicolumn{2}{|c|}{ Dimorphisme adulte avec } \\
\hline & nbre d'œufs & âge au $1^{\mathrm{er}}$ ouf & nbre d'œufs & âge au $1^{\text {er }} œ u f$ \\
\hline 1966 & $+0,13^{*}$ & $-0,02$ & $--0,52 * *$ & $+0,22 * *$ \\
\hline 1967 & $-0,03$ & $+0,28^{* *}$ & $-0,14$ & $+0,28 * *$ \\
\hline Ensemble & $+0,09$ & $+0,00$ & $-0,33^{* *}$ & $+0,24_{4} * *$ \\
\hline
\end{tabular}


Il apparaît qu'à poids égal pour les femelles, des mâles plus lourds à 8 semaines ont des sœurs de maturité sexuelle légèrement plus précoce dans la souche "Jouy ". Par contre, dans la souche M II, des mâles plus lourds adultes ont des sœurs moins précoces et pondant moins, à poids adulte fixé pour ces sœurs.

Nous avons tenté d'estimer sur une partie des données (Jouy, I966 et 1967), des corrélations génétiques à partir des moyennes de mères selon le schéma utilisé par BUVANENDRAN (1969).

Nous n'avons pu tenir compte des résultats de Ig66, où le caractère " dimorphisme sexuel à 8 semaines " présentait une composante "père "négative de la variance, ce qui enlevait d'avance toute signification aux corrélations génétiques estimées avec ce caractère.

Pour I967, la corrélation génétique du dimorphisme sexuel (basée sur les composantes " père " de la variance et de la covariance) avec l'âge au I er œuf est $-0,59$, celle avec le poids moyen des œufs $+0,48$; celle avec le nombre d'œufs n'a pu être évaluée, la composante " père " de la variance de ce caractère étant légèrement négative. Pour cette année, nos résultats s'accordent avec ceux de BuvanEndRan concernant le poids des œufs. Nos données suggèrent, d'autre part, l'existence d'une corrélation génétique négative entre dimorphisme sexuel pondéral à 8 semaines et âge au I ${ }^{\text {er }}$ œuf, corrélation de même signe que celle que nous observons au niveau phénotypique.

Au total, il est difficile de tirer une conclusion de ces estimations de corrélations génétiques, basées sur des effectifs restreints (une douzaine de pères en 1967) et présentant nécessairement une variance d'échantillonnage importante.

\section{DISCUSSION ETT CONCLUSIONS}

Parmi les corrélations phénotypiques entre la différence du poids moyen des deux sexes dans une famille et un autre caractère quantitatif, les suivantes méritent attention, vu leur degré de signification :

- A 8 semaines, corrélation positive importante entre le dimorphisme sexuel ainsi défini et le poids moyen des mâles (la corrélation avec le poids moyen des femelles étant faible et négative).

- A l'âge adulte, corrélations comparables à celles obtenues à 8 semaines, avec

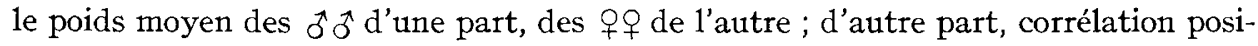
tive avec l'âge au I ${ }^{\text {er }}$ œuf et négative avec l'intensité de la ponte d'automne et d'hiver. Pour ces deux dernières corrélations, on constate que les corrélations partielles correspondantes, à poids moyen fixé pour les femelles, ont une valeur très voisine (respectivement $+0,24$ et $-0,33$ ) et que, d'autre part, la corrélation entre ces deux caractères de ponte (moyenne des sœurs) et le poids adulte moyen des frères, également à poids fixé pour les 우, a une valeur du même ordre : respectivement $+0, \mathbf{I} 4$ $(\mathrm{P}<0,0$ I $)$ et $-0,23(\mathrm{P}<0,0$ I $)$.

Le premier groupe de corrélations (entre " dimorphisme " et poids des $\sigma^{\star}{ }^{\star}$ ou des 우) représente un résultat très analogue à celui obtenu par BuvaNENDRAN (I969) sur des animaux de ro semaines. Nous devons remarquer cependant qu'il ne repré- 
sente pas par lui-même un phénomène biologique réellement indépendant; il est plutôt la traduction de l'ensemble des paramètres biométriques du poids dans chaque sexe à un âge donné.

En effet, soit $x$ le poids moyen des $\sigma^{\hat{\alpha}} \hat{\delta}, y$ celui des 우우 dans une famille de frèressœurs. Prenant $o$ pour valeur moyenne de chaque sexe dans la population dont est tiré l'échantillon observé, soient $\sigma_{x}$ et $\sigma_{y}$ les écarts-types de $x$ et $y$ et $\rho_{x y}$ le coefficient de corrélation entre $x$ et $y$ dans cette population. La première corrélation considérée, $r$, est celle entre $x-y$ et $x$. Son espérance mathématique est :

$$
\mathrm{E}(r)=\frac{\mathrm{E}(x \cdot(x-y))}{\sigma_{x} \cdot \sigma_{(x-y)}}=\frac{\sigma_{x}-\rho_{x y} \cdot \sigma_{y}}{\sqrt{\sigma_{x}^{2}+\sigma_{y}^{2}-2 \rho_{x y} \sigma_{y} \sigma_{x}}}
$$

En admettant 0,9 pour ordre de grandeur du rapport $\frac{\sigma_{y}}{\sigma_{x}}$, et une valeur de $\rho_{x y}$ comprise entre $+0,5$ et $+\mathrm{I}, \mathrm{O}$, on obtient une valeur de l'expression ci-dessus de l'ordre de $+0,5$ à $+0,6$, se rapprochant du résultat observé.

Par contre, en raisonnant de même, l'espérance mathématique du coefficient de corrélation entre dimorphisme sexuel et poids des 우 sera :

$$
\mathrm{E} \frac{(y \cdot(x-y))}{\sigma_{y} \cdot \sigma_{(x-y)}}=\frac{\rho_{x y} \sigma_{x}-\sigma_{y}}{\sqrt{\sigma_{x}^{2}+\sigma_{y}^{2}-2 \rho_{x y} \sigma_{x} \sigma_{y}}}
$$

Avec les mêmes valeurs prises pour $\rho_{x y}$ et pour $\frac{\sigma_{y}}{\sigma_{x}}$, l'expression ci-dessus sera voisine de zéro ou négative, en conformité avec les observations.

Quant aux corrélations entre dimorphisme sexuel au stade adulte et âge au I ${ }^{\text {er }}$ œuf ou intensité de ponte d'hiver, quoique peu étroites, elles paraissent intéressantes. Elles ne reflètent pas simplement une corrélation directe entre le poids adulte des poules et leurs caractéristiques pour la ponte, d'après les valeurs trouvées pour les corrélations partielles entre dimorphisme ou poids des $\widehat{o} \sigma^{\widehat{t}}$ et ponte ou précocité de leurs sœurs à poids moyen fixé pour celles-ci. Dans les familles où les $\hat{o} \hat{o}$ sont plus lourds, en valeur absolue ou relativement aux $q$,, leurs sœurs sont en moyenne moins précoces et ont une intensité de ponte un peu inférieure. Il serait intéressant de savoir si ce résultat a une portée générale ou est particulier à la population étudiée, et s'il correspond bien à une liaison de nature génétique. I'estimation trop imprécise des corrélations génétiques ne permet pas encore d'affirmer ce dernier point avec une certitude absolue, mais cela parait vraisemblable, compte tenu de l'absence d'environnement particulier commun aux $\delta^{\widehat{t}}{ }^{\widehat{\lambda}}$ et aux 우 d'une même famille depuis l'éclosion dans nos conditions d'élevage. Notre résultat suggérerait alors de tenir compte, dans la sélection par familles pour la ponte, du poids des frères, au moins dans une population telle que la nôtre. Notons que BuVANENDRAN (I969) trouve une corrélation légèrement positive avec le nombre d'œufs, mais il ne s'agit pas de la même période pour l'enregistrement de la ponte. Par contre, nous ne retrouvons pas la corrélation positive qu'il obtient avec le poids moyen des œufs ; ce dernier, il est vrai, n'est pas mesuré non plus au même stade que dans nos données. D'autres part, les corrélations données par BUVANENDRAN ne sont pas phénotypiques comme les nôtres, mais génétiques, et l'on peut s'interroger dans certains cas sur leur signification, lorsqu'elles comportent une composante " père " positive et une composante " mère " négative. 
Enfin, concernant la corrélation trouvée dans la souche Jouy entre poids des coquelets à 8 semaines et âge au I ${ }^{\text {er }}$ œuf des sœurs à poids fixé pour celles-ci, elle semble rejoindre les résultats de KINNEy et SHOFFNER (I965), quoique son niveau de signification soit moindre que dans le cas des poids au stade adulte.

Rę̧u pour publication en janvier 1973.

\section{REMERCIEMENT}

Nous remercions le docteur CocHez, Station expérimentale d'Aviculture du Magneraud, des données qu'il a mises à notre disposition pour cette recherche.

\section{SUMMARY}

\section{CORRELATIONS BETWEEN SEXUAL DIMORPHISM FOR WEIGHT AND O'THER QUANTITATIVE TRAITS IN THE FOWL,}

In two populations, we estimated the phenotypic and genetic correlations between sexual dimorphism for 8 week or adult body weight (difference between male and female mean weight) and production traits, by full sib families. A positive significant correlation with age at first egg and a negative one with fall and winter egg laying is found for adult weight sex dimorphism; it is confirmed by the partial correlation between male weight and these two traits, with fixed female weight. It would be desirable to know if this correlation exists in other populations : this information could then be used in selection for egg production.

On the other hand, weight sexual dimorphism has an important positive correlation with male weight, and a slightly negative one with female weight but these correlations do not represent by themselves a really independant phenomenon.

\section{RÉFÉRENCES BIBLIOGRAPHIQUES}

Buvanendran V., I969. The heritability and genetic correlations of sexual dimorphism for Io week weight in poultry. Brit. Poult. Sci., 10, 32x-325.

KINNEy T. B., ShoffNer R. N., 1965. Heritability estimates and genetic correiations among severa traits in a meat type poultry population. Poult. Sci., 44, 1020-1032. 